Author copy of the final (peer-reviewed) version of the article published in Nature in June 2001

Please reference to: Tommasi, A., Gibert, B., Seipold, U., Mainprice, D. (2001) Anisotropy of thermal diffusivity in the upper mantle. Nature 411, 783-786. https://doi.org/10.1038/35081046

\section{Anisotropy of thermal diffusivity in the upper mantle}

\author{
Andréa Tommasi*, Benolt Gibert*, Ulfert Seipold $†$ \& David Mainprice* \\ * Laboratoire de Tectonophysique, CNRS/Université de Montpellier II, F-34095 \\ Montpellier cedex 5, France \\ $\dagger$ GeoForschungsZentrum Potsdam, Telegrafenberg, D-14473 Potsdam, Germany
}

Heat transfer in the mantle is a key process controlling the Earth's dynamics. Upper-mantle mineral phases, especially olivine, have been shown to display highly anisotropic thermal diffusivity at ambient conditions ${ }^{1,2}$, and seismic anisotropy data ${ }^{3}$ show that preferred orientations of olivine induced by deformation ${ }^{4}$ are coherent at large scales $(>50 \mathrm{~km})$ in the upper mantle. Thus heat transport in the upper mantle should be anisotropic. But the thermal anisotropy of mantle minerals at high temperature ${ }^{1,5}$ and its relationship with deformation have not been well constrained. Here we present petrophysical modelling and laboratory measurements of thermal diffusivity in deformed mantle rocks between temperatures of 290 and $1,250 \mathrm{~K}$ that demonstrate that deformation may induce a significant anisotropy of thermal diffusivity in the uppermost mantle. We found that heat transport parallel to the flow direction is up to 30 per cent faster than that normal to the flow plane. Such a strain-induced thermal anisotropy implies that the upper-mantle temperature distribution, rheology and, consequently, its dynamics, will depend on deformation history. In oceans, resistive drag flow would result in lower vertical diffusivities in both the lithosphere and asthenosphere ${ }^{6}$ and hence in less effective heat transfer from the convective mantle. In continents, olivine orientations frozen in the lithosphere may induce anisotropic heating above mantle plumes, favouring the reactivation of pre-existing structures.

To investigate the interdependence between flow and thermal anisotropy, that is, to introduce this anisotropy in geodynamical

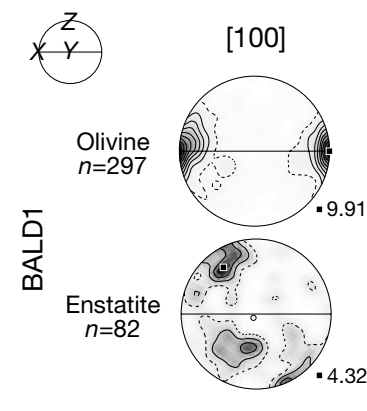

[010]
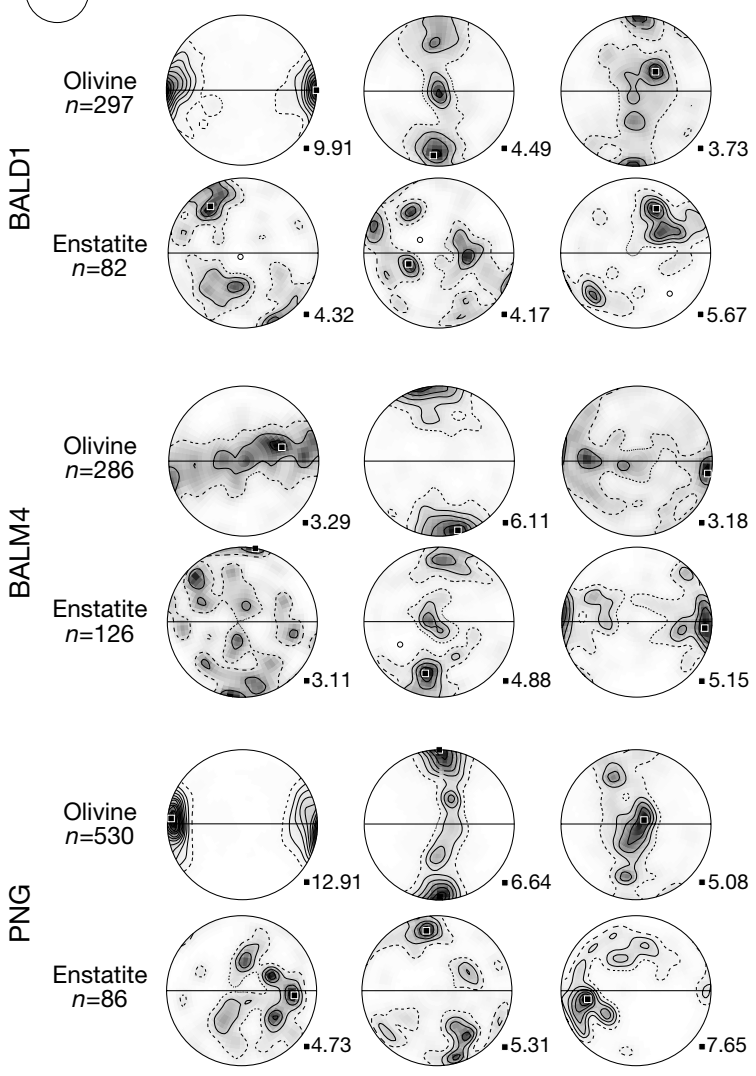

[001]

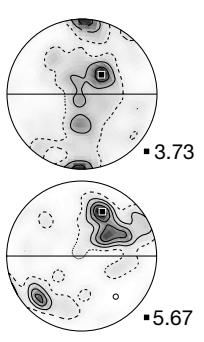

models and quantify its effects, we need to be able to predict the anisotropy of thermal diffusivity that will result from a given uppermantle flow. Polycrystal plasticity models allow the simulation of the development of olivine lattice-preferred orientations in the upper mantle ${ }^{6-9}$. If the crystallographic orientations and the singlecrystal tensor of an anisotropic physical property are known, we may estimate the three-dimensional distribution of this property in a polycrystalline aggregate by averaging the individual grain tensors ${ }^{10}$. Indeed, a strong correlation between olivine fabric and thermal diffusivity anisotropy was observed in a dunite at ambient conditions ${ }^{1}$. Temperature derivatives of the olivine thermal diffusivity tensor are nevertheless poorly constrained. Whereas thermal diffusivity data for olivine and pyroxene crystals showed a constant anisotropy independent of temperature ${ }^{1}$, more recent thermal conductivity determinations in olivine crystals ${ }^{5}$ exhibited a decrease in anisotropy with increasing temperature, similar to that observed for quartz ${ }^{11}$. Moreover, this simple petrophysical model does not consider parameters that may influence heat transport at the sample and larger scales such as a variation in thermal diffusivity at grain boundaries. These effects as well as the relationship between crystallographic orientation and anisotropy may nevertheless be constrained by comparison between model predictions and thermal diffusivity measurements on real rock samples.

We have thus determined the three-dimensional thermal diffusivity of three mantle rocks by both petrophysical modelling and laboratory measurements between 290 and 1,250 K. Two spinel lherzolites (BALM4 and BALD1) from the Balmuccia and Baldissero massifs in the Italian Alps were chosen as representativeof the subcontinental mantle and a spinel harzburgite (PNG) from the Papua New Guinea ophiolite was selected as representative of the suboceanic mantle. Modal compositions are: BALM4 (76\% olivine, 19\% enstatite, 3\% diopside and $2 \%$ spinel), BALD1 (74\% olivine, 18\% enstatite, $6 \%$ diopside and $2 \%$ spinel), and PNG (87\% olivine, $12 \%$ enstatite and $1 \%$ spinel). All samples display coarser- grained porphyroclastic textures characteristic of deformation under high-temperature conditions $(\mathrm{ml}, 100 \mathrm{mC})$ and are devoid of alteration minerals and macroscopic fractures. Olivine displays a bimodal grain size distribution: porphyroclasts range from 5 to $2 \mathrm{~mm}$, whereas recrystallized grains are smaller than 1 $\mathrm{mm}$. Pyroxenes generally form clusters that may reach up to $5 \mathrm{~mm}$. Foliation and lineation are defined by alignment of spinel crystals accompanied, in the lherzolites, by a weak shape-preferred orientation of olivine. In PNG, recrystallization by subgrain rotation leads to elongation of olivine porphyroclasts normal to the spinel lineation.

Lattice-preferred orientations (Fig. 1) are typical of peridotites deformed under high-temperature conditions, in which (010) [100] and (001)[100] are the dominant slip systems for olivine9. BALD1 and PNG display a strong concentration of olivine [100] axes subparallel to the lineation (flow direction) and a distribution of both [010] and [001] axes in a plane normal to the lineation, with secondary concentrations normal and parallel to the foliation (flow plane), respectively. BALM4, on the other hand, is characterized by a planar distribution of [100] and [001] along the foliation and a [010] concentration normal to it. Enstatite preferred orientations are weaker. BALM4 and PNG are characterized by a concentration of the main glide direction [001] close to the lineation and a weak concentration of [010] at high angle to the foliation. BALD1 enstatite preferred orientation is oblique to the foliation. Diopside displays very weak preferred orientations.

Figure 1 Olivine and enstatite lattice-preferred orientations for the studied samples. Lower hemisphere equal-area projection, $n$ measurements, contour intervals are at multiples of a uniform distribution. Inverse-log shading varies from white (minimum density) to black (maximum density, also indicated by a solid square). Full line marks the foliation ( $X-Y$ plane); lineation ( $X$ direction) is horizontal. 
Petrophysical modelling using olivine and pyroxene thermal diffusivity tensors determined by spectroscopic methods at ambient conditions $^{2}$ indicates that all samples display a significant anisotropy of thermal diffusivity (between 17.5 and 28.6\%; Fig. 2). Thermal diffusivity is maximum parallel to the olivine [100] concentration, that is, parallel to the lineation ( $X$ direction), and minimum parallel to the $[010]$ concentration, that is, normal to the foliation ( $Z$ direction). Anisotropy depends on the strength of olivine lattice-preferred orientation; PNG displays the strongest lattice-preferred orientation and the most anisotropic behaviour. Thermal anisotropy also depends on the pyroxene content, which tends to weaken the anistropy; this effect is enhanced when olivine and estatite lattice-preferred orientations are uncorrelated, as in BALD1. In addition, thermal diffusivity of olivine varies more between [010] and [001] directions than between [001] and [100]. Thus the dispersion of [010] and [001] in a plane normal to the lineation Y-Z plane) showed by BALD1 significantly weakens its anisotropy, whereas the dispersion of [100] and [001] in the foliation (X-Y plane) displayed by BALM4 only slightly reduces its maximum thermal diffusivity. Similar patterns, but higher anisotropies (between 23.9 and $38.4 \%$ ), are predicted if more anisotropic olivine and enstatite diffusivity tensors ${ }^{1}$ are used.

Modelling suggests that, at ambient conditions, upper-mantle rocks retain one-third to half of the olivine single crystal anisotropy. Is this anisotropy preserved at upper-mantle conditions? Does dispersion at grain boundaries affect the thermal diffusivity tensor? In order to answer these questions, we measured the thermal diffusivity of our samples at ambient pressure and temperatures between 290 and 1,250 K. Six measurements were performed parallel to the lineation (X direction, [100] concentration, for which the highest diffusivity is predicted), four normal to the flow plane ( $\mathrm{Z}$ direction, [010] concentration), and two in the flow plane, but normal to the lineation (Y direction, [001] concentration). These measurements (Fig. 3) show a significant anisotropy of thermal diffusivity that remains roughly constant over the entire temperature range, in agreement with high-temperature thermal diffusivity data on olivine and pyroxene crystals ${ }^{1}$. Measured anisotropies (21\% for BALM4 and 12\% for BALD1) are consistent with predictions of petrophysical models using data from ref. 2 (Fig. 2). The higher anisotropy (31\%) previously observed in a dunite ${ }^{1}$ may be explained by the lower pyroxene content and very strong crystallographic orientation ${ }^{12}$ of that sample (similar to PNG, for which $28 \%$ of anisotropy was predicted).
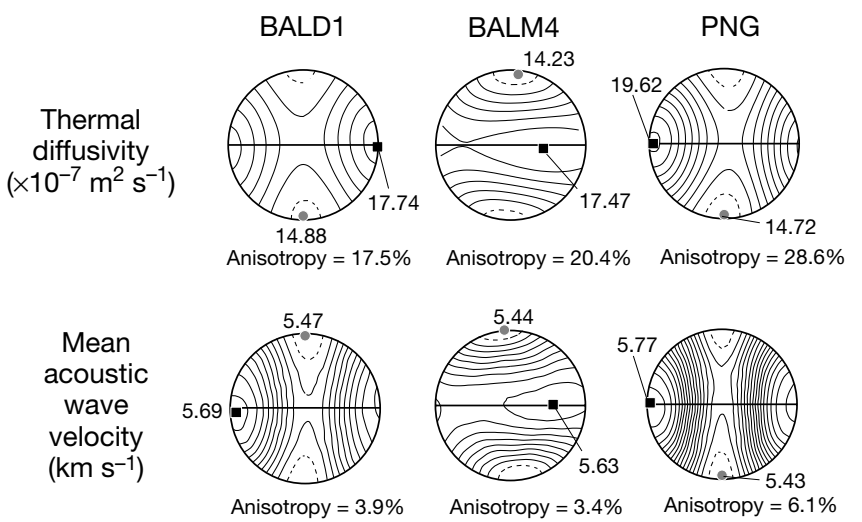

Figure 2 Modelled three-dimensional thermal diffusivity and mean acoustic wave velocity distributions. Voigt-Reuss-Hill averages calculated from crystallographic orientation data and thermal diffusivity and elastic constants tensors for olivine and enstatite at ambient conditions ${ }^{2,27,28}$. As its full thermal diffusivity tensor is not known, diopside is assimilated to enstatite. $V_{\text {mean }}=3 /\left(1 / V_{p}+1 / V_{S 1}+1 / V_{S 2}\right)$. Lower hemisphere equalarea projections, contours at $10^{-7} \mathrm{~m}^{2} \mathrm{~s}^{-1}$ and $0.02 \mathrm{~km} \mathrm{~s}^{-1}$ intervals, respectively. Full line marks the foliation ( $X-Y$ plane); lineation ( $X$ direction) is horizontal.
Maximum and minimum thermal diffusivities are coherent from sample to sample and their relative magnitudes match model predictions very well: $K_{\mathrm{PNG}-\mathrm{X}}>K_{\mathrm{BALD} 1-\mathrm{X}} \approx K_{\mathrm{BALM} 4-\mathrm{X}}>K_{\mathrm{BALD1}-\mathrm{Y}}>$ $K_{\text {BALM4-Z }}$. Measured thermal diffusivities at $290 \mathrm{~K}$ are, however, systematically lower by $20 \%$ than model predictions; this difference may be attributed to isotropically distributed lattice imperfections and microfractures in the samples. Indeed, thermal diffusivity measurements in crystalline rocks under increasing pressure conditions generally show a fast, non-linear increase in thermal diffusivity up to $200 \mathrm{MPa}$ associated with closure of microfractures ${ }^{13,14}$. Thus petrophysical models using olivine and pyroxene thermal diffusivity tensors from ref. 2, our experimental constraints on the temperature-derivatives of the olivine and pyroxene thermal diffusivity tensors, as well as a weak linear dependence on pressure ${ }^{2,15}$, should provide good estimates of the upper-mantle thermal diffusivity.

Relative contributions of phonon conduction and radiative transfer to the total diffusivity may be inferred from the temperature dependence of thermal diffusivity. Up to $600 \mathrm{~K}$, thermal diffusivity is inversely proportional to temperature, suggesting that scattering of heat-conducting phonons by lattice imperfections and phonon-phonon interactions controls heat transport ${ }^{19}$.At higher temperature, deviation of this behaviour may be ascribed to the onset of radiative heat transfer. Above $900 \mathrm{~K}$, this contribution (about $15 \%$ of the total diffusivity) is restrained by development of oxide films along grain boundaries (observed optically in thin sections of the measured cores). Thus lattice conduction is the dominant heat transfer mechanism over the entire temperature range of our measurements. Lattice conduction should also prevail in the Earth's upper mantle, as suggested by analysis of infrared absorption data ${ }^{20}$. Yet, although it is minor, the radiative contribution at high temperature may have been underestimated in our experiments.

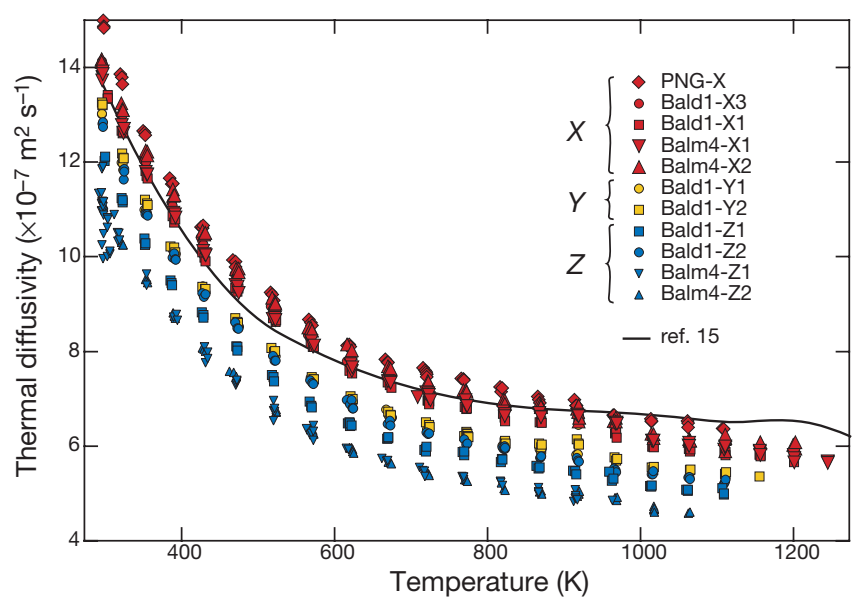

Figure 3 Thermal diffusivity as a function of temperature as measured in oriented cores of PNG (diamonds), BALD1 (circles and squares), and BALM4 (triangles). Red symbols mark measurements parallel to the [100] axis maximum $(X$ direction $=$ lineation), blue symbols indicate measurements parallel to the [010] axis maximum $(Z$ direction $=$ normal to the foliation), and yellow symbols show measurements in the $Y$ direction for BALD1. At each temperature, three successive measurements are performed in order to check data reproducibility. High dispersion in BALM4-Z1 low-temperature data results from electronic noise, eliminated in subsequent measurements. Error bars for individual measurements (not plotted) are of $2 \%$. Relatively high thermal diffusivities displayed by BALD1 in the $Z$ direction result from the dispersion of [010] and [001] in the $Y-Z$ plane (compare Fig. 1). For comparison, the full black line represents thermal diffusivity data for a fine-grained isotropic olivine polycrystal at $100 \mathrm{MPa}^{15}$. 
Heat transfer by phonons depends essentially on phonon velocity and mean free path ${ }^{21}$. Anisotropy of phonon velocities, which may be approximated by the anisotropy of acoustic velocities ${ }^{1}$, is significantly lower than the anisotropy of thermal diffusivity (Fig. 2). Thus the observed thermal diffusivity anisotropy largely results from anisotropy of the phonon mean free path. This latter is controlled by interactions with lattice imperfections and phonon- phonon collisions. At high temperature, however, the contribution of phonon-phonon interactions is dominant. Preservation of a constant anisotropy over the entire temperature range suggests therefore that phonon mean free path and, hence, anisotropy are controlled by phonon-phonon collisions, which are more frequent along the [010] direction. Indeed, linear thermal expansion coefficients for olivine, which are also related to the anharmonicity of lattice potential, display a similar anisotropy, $\alpha_{[100]}<\alpha_{[001]}<\alpha_{[010]}$ up to $2,000 \mathrm{~K}$ (ref. 22 ).

Through the association of petrophysical modelling and thermal diffusivity measurements on textured upper-mantle rocks, we show that deformation may induce a significant thermal diffusivity anisotropy in the uppermost mantle: heat transport parallel to the olivine [100] axes concentration (flow direction) is up to $30 \%$ faster than normal to the flow plane ([010] concentration). Moreover, because the anisotropy does not depend on temperature, it might be preserved under higher-temperature, asthenospheric conditions. Existence of a large-scale, strain-induced thermal anisotropy in the upper mantle implies that the temperature distribution, rheol- ogy, and, hence, the upper-mantle dynamics depend on its defor- mation history. Olivine orientations frozen in the continental lithosphere may modify plume-lithosphere interactions. Enhanced thermal diffusivity along lithospheric-scale wrench zones-that is, parallel to the olivine [100] preferred orientation-may lead to anisotropic heating of the lithosphere, favouring the reactivation of these structures during continental break-up ${ }^{23,24}$. Such a control of the pre-existing lithospheric structure on the propagation of a thermal anomaly may be inferred, for instance, from tomographic images of the east African rift $^{25}$. In oceanic domains, deformation by resistive drag of the sublithospheric mantle leads to vertical orientation of olivine [010] axes in the lithosphere and asthenosphere6, which will result in reduced vertical diffusivity and hence in less effectiv heat transfer from the convective mantle. Reduced diffusivity across oceanic plates may also lead to slower heating of subducting slabs. Interactions between flow and anisotropy are nevertheless complex and a full investigation of the effect of an anisotropic thermal diffusivity on mantle convection may only be accomplished by integrating this strain-induced anisotropy in geodynamical models.

\section{Acknowledgements}

We thank C. Karger for participation in the measurements, and M. Ferrero and F. Boudier for providing the samples from Baldissero and Papua New Guinea, respectively. The Laboratoire de Tectonophysique's EBSD system was funded by the CNRS/INSU, Université of Montpellier II, and NSF project "Anatomy of an archean craton". This work was supported by the CNRS/INSU programme "Action Thématique Innovante".

Correspondence should be addressed to A.T.

(e-mail: deia@dstu.univ-montp2.fr)

\section{Methods}

\section{Lattice-preferred orientation measurements}

Crystallographic orientations were determined on a scanning electron microscope (JEO JSM 5600) by indexation of electron back-scattered diffraction (EBSD) patterns generated by interaction of a vertical incident electron beam with a carefully polished thin section tilted at $70^{\circ}$. The pattern is projected onto a phosphor screen and recorded by a low-light high-resolution charge-coupled device (CCD) camera. The image is then digitally processed and indexed in terms of crystal orientation using the CHANNEL+ software from HKL Technology.

\section{Thermal diffusivity measurements}

For each sample, thermal diffusivity of one (PNG) to six (BALD1) oriented cores of 43 $\mathrm{mm}$ length and $27 \mathrm{~mm}$ diameter was determined using a finite pulse method ${ }^{26}$. The energy pulse $(3 \mathrm{~s})$ is generated at the core axis by a Nichrome heater. It propagates radially and is recorded by a Chromel/Alumel thermocouple placed at a distance $d$ (6-7 $\mathrm{mm}$ ) from the core axis. The temperature response is measured every $0.1 \mathrm{~s}$ during $40 \mathrm{~s}$. From the measured temperature curve, the half-time value $t_{\mathrm{H}}$, that is, the time span to reach half of the maximum temperature rise, is evaluated. Thermal diffusivity $K$ is then calculated as $K=d^{2} /\left(10.77 t_{\mathrm{H}}-16.55\right)$. A thick-walled pyrophyllite container prevents thermal con- vection around the sample and a drastic step in thermal properties at sample boundaries. The entire assemblage is fitted in a three-zone furnace. At each temperature, three successive measurements are performed in order to check data reproducibility. Moreover, for samples BALM4 and BALD1, two series of measurements (two cores) were performed in each direction.

1. Kobayashi, Y. Anisotropy of thermal diffusivity in olivine, pyroxene, and dunite. J. Phys. Earth 22, 359-373 (1974).

2. Chai, M., Brown, J. M. \& Slutsky, L. J. Thermal diffusivity of mantle minerals. Phys. Chem. Minerals 23, 470-475 (1996)

Silver, P. G. Seismic anisotropy beneath the continents: Probing the depths of geology. Аnпu. Rev. Earth Planet. Sci. 24, 385-432 (1996).

4. Nicolas, A. \& Christensen, N. I. in Composition, Structure and Dynamics of the LithosphereAsthenosphere System (eds Fuchs, K. \& Froidevaux, C.) 111-123 (American Geophysical Union, Washington DC, 1987)

5. Schärmeli, G. H. in High-Pressure Researches in Geosciences (ed. Schreyer, W.) 349-373 (E. Schweizerbart'sche, Stuttgart, 1982).

6. Tommasi, A. Forward modeling of the development of seismic anisotropy in the upper mantle. Earth Planet. Sci. Lett. 160, 1-13 (1998).

7. Chastel, Y. B., Dawson, P. R., Wenk, H.-R. \& Bennet, K. Anisotropic convection with implications for the upper mantle. J. Geophys. Res. 98, 17757-17771 (1993).

8. Blackman, D. K. et al. Teleseismic imaging of subaxial flow at mid-ocean ridges: travel-time effects of anisotropic mineral texture in the mantle. Geophys. J. Int. 127, 415-426 (1996).

9. Tommasi, A., Mainprice, D., Canova, G. \& Chastel, Y. Viscoplastic self-consistent and equilibriumbased modeling of olivine lattice preferred orientations. Implications for upper mantle seismic anisotropy. J. Geophys. Res. 105, 7893-7908 (2000).

10. Mainprice, D. \& Humbert, M. Methods of calculating petrophysical properties from lattice preferred orientation data. Surv. Geophys. 15, 575-592 (1994).

11. Kanamori, H., Fuii, N. \& Mizutani, H. Thermal diffusivity measurement of rock-forming minerals from $300^{\circ} \mathrm{K}$ to $1100^{\circ} \mathrm{K}$. J. Geophys. Res. $73,595-605$ (1968).

2. Kasahara, J., Suzuki, I., Kumazawa, M., Kobayashi, Y. \& Iida, K. Anisotropism of P wave in dunite. Zisin (J. Seismol. Soc. Jpn) 21, 222-228 (1968).

13. Horai, K. \& Susaki, J. The effect of pressure on the thermal conductivity of silicate rocks up to $12 \mathrm{kbar}$. Phys. Earth Planet. Inter. 55, 292-305 (1989).

14. Seipold, U., Mueller, H. J. \& Tuisku, P. Principle differences in the pressure dependence of thermal and elastic properties of crystalline rocks. Phys. Chem. Earth 23, 357-360 (1998).

15. Katsura, T. Thermal diffusivity of olivine under upper mantle conditions. Geophys. J. Int. 122, 63-69 (1995).

16. Shankland, T. J., Nitsan, U. \& Duba, A. G. Optical absorption and radiative heat transport in olivine at high temperature. J. Geophys. Res. 84, 1603-1610 (1979).

17. Schatz, J. F. \& Simmons, G. Thermal conductivity of Earth materials at high temperatures. J. Geophys, Res. 77, 6966-6983 (1972).

18. Holt, J. B. Thermal diffusivity of olivine. Earth Planet. Sci. Lett. 27, 404-408 (1975).

19. Seipold, $U$. Temperature dependence of thermal transport properties of crystalline rocks-general law. Tectonophysics 291, 161-171 (1998)

20. Hofmeister, A. M. Mantle values of thermal conductivity and the geotherm from phonon lifetimes. Science 283, 1699-1706 (1999).

21. Klemens, P. G. Thermal conductivity and lattice vibrational modes. Solid State Phys. 7, 1-98 (1958).

22. Bouhifd, M. A., Andrault, D., Fiquet, G. \& Richet, P. Thermal expansion of forsterite up to the melting point. Geophys. Res. Lett. 23, 134-1136 (1996)

23. Vauchez, A., Barruol, G. \& Tommasi, A. Why do continents break up parallel to ancient orogenic belts? Terra Nova 9, $62-66$ (1997).

24. Tommasi, A. \& Vauchez, A. Continental rifting parallel to ancient collisional belts: An effect of the mechanical anisotropy of the lithospheric mantle. Earth Planet. Sci. Lett. 185, 199-210 (2001).

25. Achauer, U. \& KRISP working group. New ideas on the Kenya rift based on the inversion of the combined dataset of the 1985 and 1989/90 seismic tomography experiments. Tectonophysics 236, 305329 (1988).

26. Seipold, U. Simultaneous measurements of thermal diffusivity and thermal conductivity under high pressure using thermal pulse of finite length. High Temp.-High Press. 20, 609-613 (1988).

27. Abramson, E. H., Brown, M., Slutsky, L. J. \& Zaug, J. The elastic constants of San Carlos olivine up to 17 GPa. J. Geophys. Res. 102, 12252-12263 (1997).

28. Duffy, T. S. \& Vaughan, M. T. Elasticity of enstatite and its relationships to crystal structure. J. Geophys. Res. 93, 383-391 (1988). 